\title{
Long-term changes in insulin action and insulin secretion associated with gain, loss, regain and maintenance of body weight
}

\author{
C. Weyer, K. Hanson, C. Bogardus, R. E. Pratley \\ Clinical Diabetes and Nutrition Section, National Institute of Diabetes and Digestive and Kidney Diseases, \\ National Institutes of Health, Phoenix, Arizona, USA
}

\section{Abstract}

Aims/hypothesis. We aimed to quantify changes in insulin action and insulin secretion associated with long-term gain, loss, regain, and maintenance of body weight in subjects with normal (NGT) or impaired (IGT) glucose tolerance.

Methods. Insulin action (hyperinsulinaemic clamp) and insulin secretion (intravenous glucose challenge) were measured longitudinally in 209 Pima Indians [body weight $94.4 \pm 22.8 \mathrm{~kg}$ (means $\pm \mathrm{SD}$ ) 89 women/ 120 men, 151 NGT/58 IGT], who either lost $(n=110)$ or gained $(n=99)$ weight $(-23 \%$ to $+29 \%$ ) over $2.6 \pm 2.0$ years. Insulin action and insulin secretion were reassessed on a third occasion in 33 subjects who lost at least $5 \%$ body weight over $1.5 \pm 0.8$ years and then either regained or maintained weight over the subsequent $1.8 \pm 1.1$ years.

Results. There was a linear negative relation between changes in body weight and changes in insulin-stimulated glucose disposal in subjects with normal glucose tolerance $(r=-0.51, p<0.0001)$ and impaired glucose tolerance $(r=-0.54, p<0.0001)$. In contrast, changes in the acute insulin response were positively related to weight changes in subjects with normal glucose tolerance $(r=+0.26, p<0.005)$ but negatively in those with impaired glucose tolerance $(r=-0.51$, $p<0.0001)$. Improvements in insulin action after an average of $10 \%$ weight loss were lost with weight regain but largely preserved with weight maintenance. Conclusion/interpretation. Improvements in insulin action are proportional to the amount of weight loss, similar in magnitude to the impairment in insulin action with weight gain, preserved with long-term weight maintenance and similar between subjects with normal and with impaired glucose tolerance. Weight gain could, however, have more detrimental effects in people with impaired glucose tolerance, in whom insulin secretion decreases rather than increases to compensate for the decreased insulin action. [Diabetologia (2000) 43: 36-46]

Keywords Weight loss, weight gain, weight regain, weight maintenance, weight cycling, insulin resistance, acute insulin response, disposition index, glucose clamp, obesity.
Obesity [1-3] and weight gain [3-5] are independent risk factors for the development of Type II (non-insulin-dependent) diabetes mellitus and many obese

Received: 10 May 1999 and in revised form: 2 August 1999

Corresponding author: C. Weyer, MD, Clinical Diabetes and Nutrition Section, National Institutes of Health, $4212{\mathrm{~N} 16^{\text {th }}}$ Street. Rm. 5-41, Phoenix, AZ, 85016 USA

Abbreviations: AIR, Acute insulin response; EMBS, estimated metabolic body size; M-high, insulin-stimulated glucose disposal at high dose insulin infusion; M-low, insulin-stimulated glucose disposal at low dose insulin infusion. people are characterized by several prediabetic abnormalities such as impaired glucose tolerance, hyperinsulinaemia and insulin resistance [6-8]. Clinically, it is well-known that glucose tolerance typically improves with weight loss and deteriorates with weight gain and weight reduction is thus commonly recommended to overweight people with normal (NGT) or impaired (IGT) glucose tolerance.

Although the beneficial effects of weight loss on glucose tolerance have been well documented in numerous intervention studies [9-21], several aspects relevant to the use of weight reduction as a therapeu- 
tic means remain to be clarified. These include questions as to the underlying changes in insulin action and insulin secretion and their relative contributions to the changes in glucose tolerance, the variability among people in their metabolic response to changes in body weight, particularly among those with NGT compared with IGT and the long-term changes in insulin action and insulin secretion associated with weight maintenance compared with regain after initial weight loss.

Results from glucose clamp studies suggest that much of the improvement in glucose tolerance with weight loss is attributable to an improvement in insulin action [9-17]. Glucose tolerance is, however, a function not only of insulin action but also of insulin secretion $[22,23]$ and the effect of weight change on glucose tolerance will thus depend upon changes in both of these measures. The effects of weight loss and weight gain on insulin secretion have been less well investigated [18-20], particularly in subjects with IGT in whom defects in insulin secretion could already be present at baseline [6].

The quantitative relation between changes in body weight and changes in metabolic function, especially in the long term, also requires further clarification. In particular, it would be valuable to know whether such relations are linear, i.e. whether greater weight loss will result in proportionally greater metabolic improvements. Furthermore, it is important to gain insight into the interindividual variability in the metabolic responses to weight change and to identify possible determinants underlying such variation.

Finally, there is limited information as to what extent initial improvements in glucose tolerance and insulin action after weight loss can be preserved over the long term if weight is successfully maintained and to what extent they diminish if weight is regained [21]. The latter aspect is of particular interest as most people regain weight and it has been proposed that repeated weight loss and regain ("weight cycling") has adverse effects on body fat distribution [24-26], glucose tolerance [26] and risk of diabetes [27].

The above questions can only be addressed in detailed longitudinal studies, in which glucose tolerance, insulin action and insulin secretion are repeatedly measured over several years before and after gain, loss, regain, and maintenance of body weight. In this study, we aimed to further examine these relations using data from a comprehensive longitudinal study $[28,29]$ in the Pima Indians of Arizona, a population with a very high prevalence of obesity and Type 2 diabetes [2].

\section{Subjects and methods}

Subjects. All subjects were Native Americans from the Gila River Indian Community near Phoenix, Arizona. They were between 18 and 50 years of age, healthy according to a physical examination and routine laboratory tests and did not smoke or take medications at the time of the study. Subjects were selected from an ongoing longitudinal study of the determinants of Type 2 diabetes [28, 29]. In this study, subjects are admitted to the metabolic ward of the Clinical Diabetes and Nutrition Section of the National Institutes of Health in Phoenix for approximately 8-10 days, during which they are fed a weight-maintaining diet $(50 \%, 30 \%$ and $20 \%$ of daily calories provided as carbohydrate, fat and protein, respectively) and abstain from strenuous exercise. After at least 3 days on the diet, subjects undergo a series of tests for the assessment of body composition, glucose tolerance, insulin action and insulin secretion (see below). Subjects are then asked to return to the metabolic ward at approximately annual intervals for repetition of the entire series of tests at each follow-up admission. Of the 443 subjects enrolled into the study, 272 had been studied on at least two occasions. Of these, all subjects $(n=209)$ were included who were non-diabetic at baseline and at least one subsequent admission according to both the 1985 World Health Organisation (WHO) [30] and the 1997 American Diabetes Association (ADA) [31] diagnostic criteria. Based on the results of a 75-g oral glucose tolerance test [30], 151 of the 209 subjects had normal (NGT) and the remaining 58 had impaired (IGT) glucose tolerance at baseline. If subjects had been studied more than twice while non-diabetic and had lost weight at more than one follow-up visit, then the results of the follow-up visit with the lowest body weight were included in the analysis (weight losers, $n=110$ ). If subjects had a steady increase in body weight over several admissions, then the results of the follow-up visit with the greatest body weight were included (weight gainers, $n=99$ ). In a first analysis, the relation between changes in fasting and 2-h glucose and insulin concentrations, insulin action and insulin secretion (see below) and the change in body weight were assessed in the entire study cohort of 209 subjects. For a second analysis, all subjects in the weight loss group with either NGT or IGT were included, who had lost at least $5 \%$ of body weight (on average $\sim 10 \%$ ) and who had been studied on a third occasion at which they had either maintained $( \pm<4 \%$ change from the reduced body weight, $n=15)$ or regained $(50-150 \%$ of the initial weight loss, $n=18$ ) body weight. The results of these two groups were compared to those of a control group of people who had been weight stable $( \pm<4 \%$ change from the initial body weight) throughout three consecutive visits over a similar time period $(n=20)$. All subjects in this second analysis were non-diabetic on each of the three occasions.

The study protocol was approved by the Institutional Review Board of the National Institute of Diabetes and Digestive and Kidney Diseases and by the Tribal Council of the Gila River Indian Community and all subjects provided written informed consent before participating.

Body weight and composition. Total body weight was measured using a precision scale (ACME San Leandro, Calif., USA) and corrected for the weight of the hospital gown. Body composition was estimated by underwater weighing with simultaneous determination of residual lung volume by helium dilution [32] or by total body dual energy x-ray absorptiometry (DPX-L; Lunar Radiation Corp., Madison, Wis., USA) [33]. Per cent body fat, fat mass and fat-free mass were calculated as described [34]. A previously derived conversion equation [35] was used to make measurements similar between 
the two methods. Waist and thigh circumferences were measured at the umbilicus and the gluteal fold in the supine and standing position, respectively and the waist-to-thigh ratio (WTR) was calculated as an index of body fat distribution.

Oral glucose tolerance test. After a 12-h overnight fast, subjects underwent a 75-g oral glucose tolerance test [30]. Plasma samples were drawn at baseline and after 30 and 120 min for determination of plasma glucose and insulin concentrations. The incremental 30-min insulin concentration, adjusted for the 30min glucose concentration in a multiple regression, was calculated as a measure of insulin secretion. Plasma glucose concentrations were determined by the glucose oxidase method (Beckman Instruments, Fullerton, Calif., USA) and plasma insulin concentrations by radioimmunoassays, using either the Herbert modification [36] of the method of Yalow and Berson [37] or an automated analyser (Concept 4, ICN, Costa Mesa, Calif., USA) (12\% cross-reactivity with proinsulin).

Two-step hyperinsulinaemic euglycaemic glucose clamp. Insulin action was assessed at physiologic and supraphysiologic insulin concentrations during a two-step hyperinsulinaemic euglycaemic glucose clamp as described [28, 29]. In brief, after an overnight fast, a primed continuous intravenous insulin infusion was given for $100 \mathrm{~min}$ at a constant rate of $40 \mathrm{mU}$ per square meter body surface area per min (low dose), followed by a second $100 \mathrm{~min}$ infusion at a rate of $400 \mathrm{mU}$ per square meter body surface area per min (high dose). These infusions achieved steady state plasma insulin concentrations of $147 \pm 42 \mu \mathrm{U} / \mathrm{ml}$ and $2222 \pm 597 \mu \mathrm{U} / \mathrm{ml}$ (means \pm SD), respectively. Plasma glucose concentrations were maintained at approximately $5.0 \mathrm{mmol} / \mathrm{l}$ with a variable infusion of a $20 \%$ glucose solution (mean $5.2 \pm 0.01 \mathrm{mmol} / \mathrm{l}$, coefficient of variation $2.3 \pm 0.03 \%)$. The rate of insulin-stimulated glucose disposal (M) was calculated for the last $40 \mathrm{~min}$ of the low-dose (Mlow) and high dose (M-high) insulin infusion [28, 29] and normalized to estimated metabolic body size $(\mathrm{EMBS}=$ fat-free mass $+17.7 \mathrm{~kg}$ ) [38].

Intravenous glucose tolerance test. On a separate day, after a 12 -h overnight fast, insulin secretion was measured in response to a 25-g intravenous glucose bolus as described [39]. The acute insulin response (AIR) to intravenous glucose was calculated as the average incremental plasma insulin concentration from the third to the fifth minute after the glucose bolus [39].

Disposition index. The product of AIR and M-low was calculated as a combined measure of insulin secretion and insulin action, in the following referred to as the disposition index as suggested previously [22].

Statistical analyses. Statistical analyses were done using the procedures of the SAS Institute [40]. Results are given as means \pm SD in the text and tables and as means \pm SEM in Figure 3.

Analysis on the effects of weight loss and weight gain. The baseline characteristics of subjects with NGT compared with IGT and of the weight loss compared with the weight gain groups were compared using general linear regression models with simultaneous adjustment for age and sex. Changes in anthropometric and metabolic variables accompanying the change in body weight were tested for statistical significance using paired $t$ tests. The relations between metabolic changes and the percent changes from baseline in body weight, fat mass and fatfree mass were assessed by linear regression analysis with calculation of the Pearson correlation coefficient. The changes in
M-low and AIR predicted for a $10 \%$ weight loss and $10 \%$ weight gain in the NGT and IGT groups were calculated from the respective regression equations. These longitudinal changes were superimposed onto the cross-sectional relation of AIR and M-low as assessed in 277 Pima Indians with NGT in our database. Stepwise and general linear regression models were used to assess the determinants of changes in the 2-h glucose concentration $(\Delta 2$-h glucose $)$, in insulin action ( $\Delta$ M-low) and in insulin secretion ( $\Delta$ AIR) and the percentage of variance in these changes that can be explained by the respective determinants $\left(r^{2}\right)$.

Analysis of the effect of weight regain compared with weight maintenance. Baseline characteristics were compared between the weight stable, weight regain and weight maintenance groups using general linear regression models with simultaneous adjustment for age and sex. The changes in anthropometric and metabolic variables within each group were tested for statistical significance using paired $t$ tests (changes between the three steps) and repeated measures analysis of variance (overall time effect). Between group comparisons were made using general linear regression models (changes between the three steps) and repeated measures analysis of variance (overall time $\times$ group effect) with additional adjustment for age, sex and follow-up duration.

\section{Results}

Effect of weight gain and weight loss. The physical characteristics and the anthropometric and metabolic changes of the weight losers and weight gainers are summarized in Table 1. Subjects with IGT were older $(+2.7 \pm 0.9$ years, $p<0.01)$ and heavier $(+8.2 \pm 3.6$ $\mathrm{kg}, p<0.05)$ at baseline than those with NGT, had higher fasting and 2-h glucose and insulin concentrations (all $p<0.0001$ ), and lower M-low, M-high (both $p<0.001)$ and AIR $(p=0.08)$. Age and body weight at baseline were lower in the weight gainers than in the weight losers in both the NGT and IGT groups. In the NGT group, insulin action at baseline was higher in the weight gainers than in the weight losers (Table 1).

Weight loss in both the NGT and IGT group was accompanied by significant reductions in per cent body fat, fat mass, fat-free mass and in the fasting glucose and insulin concentrations. Both M-low and Mhigh increased with weight loss in the two groups whereas changes in the acute insulin response (AIR) and in the incremental 30-min insulin concentration were not significant (Table 1). Conversely, weight gain was accompanied by increases in per cent body fat, fat mass, fat-free mass and the fasting glucose and insulin concentrations. In the NGT group, Mlow and M-high decreased with weight gain, whereas the AIR and the incremental 30-min insulin concentration did not change. In contrast, in the IGT group, there were no significant changes in M-low and Mhigh with weight gain but both the AIR and the incremental 30-min insulin concentration decreased (Table 1 . 
Table 1. Physical characteristics, anthropometric and metabolic changes in 209 Pima Indians with either normal (NGT, $n=151)$ or impaired (IGT, $n=58$ ) glucose tolerance at baseline who either lost or gained weight over an average of $2.6 \pm 2.0$ years

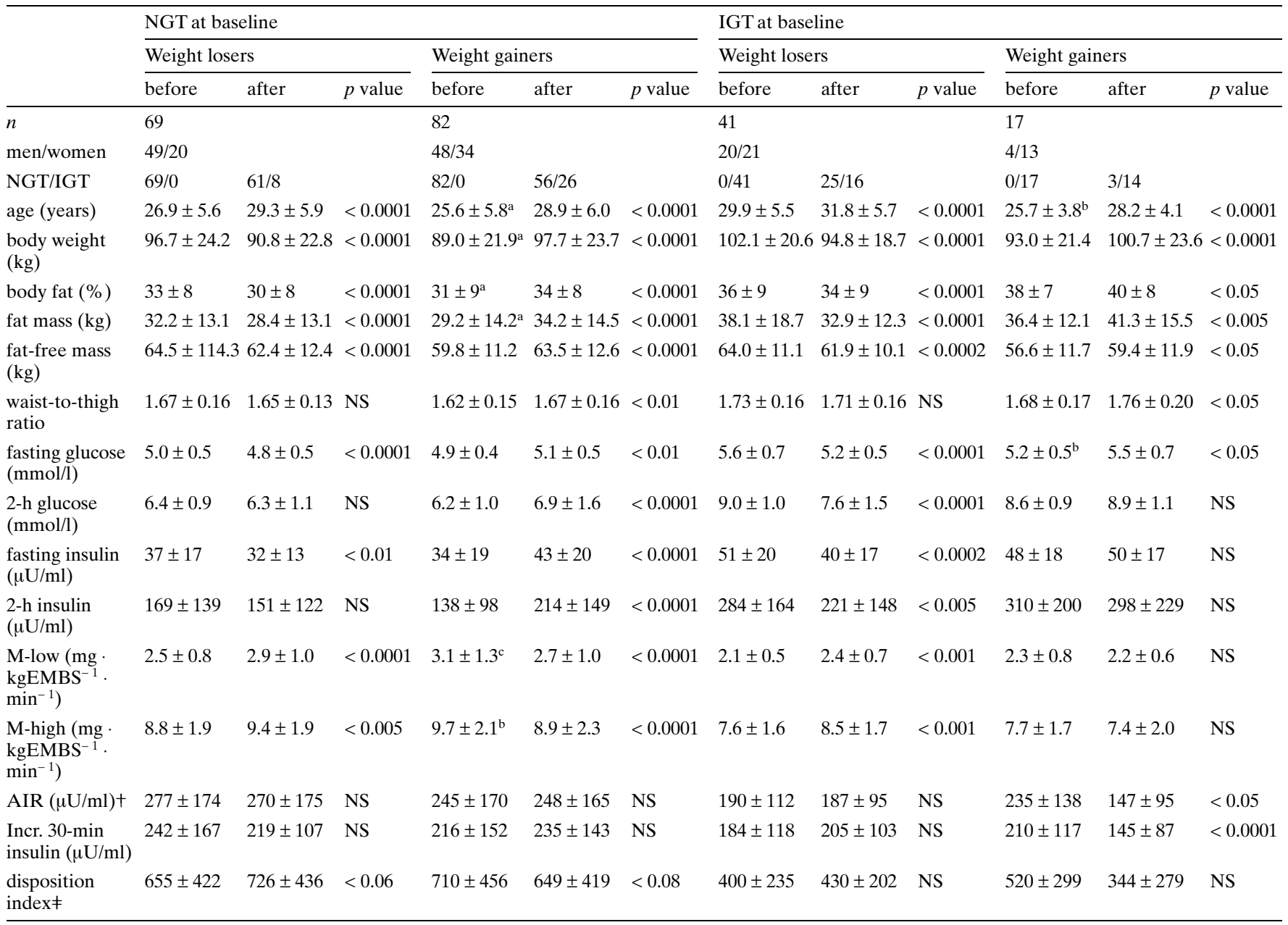

Means \pm SD. The $p$ values refer to the changes in each group over time (paired $t$ test) whereas the asterisks in the weight gainers columns indicate significant differences to the weight losers groups at baseline $\left({ }^{\mathrm{a}} p<0.05,{ }^{\mathrm{b}} p<0.01,{ }^{\mathrm{c}} p<0.001\right.$, adjusted for age and sex). +AIR was assessed in 126 of the 151 indivi-

The changes in fasting and 2-h glucose ( $\Delta$ 2-h glucose) and insulin concentrations were all negatively related to the changes in body weight ( $\Delta$ weight), fat mass ( $\Delta$ fat mass), and fat-free mass ( $\Delta$ fat-free mass) in both the NGT and IGT groups (Table 2, Fig. 1). In multiple regression analyses, these changes and all metabolic changes described subsequently, were more strongly related to $\Delta$ weight than to $\Delta$ fat mass or $\Delta$ fat-free mass. In the entire group of 209 subjects, $17 \%$ of the variance in $\Delta 2$-h glucose was explained by $\Delta$ weight. Another $17 \%$, i.e. a total of $34 \%$ of the variance in $\Delta 2$-h glucose was explained by the changes in M-low ( $\Delta$ M-low), in AIR ( $\Delta$ AIR) and by the initial glucose tolerance status, all were significant additional determinants, independent of $\Delta$ weight. Thus, a significant effect of $\Delta$ weight on $\Delta 2$-h glucose persisted, even after adjustment for $\Delta \mathrm{M}$-low and $\Delta$ AIR. duals with NGT and in 54 of the 58 subjects with IGT and multiplied with M-low to obtain the disposition index (₹ $\mu \mathrm{U} \cdot \mathrm{mg} \cdot \mathrm{ml}^{-1} \cdot \mathrm{kgEMBS}^{-1} \cdot \mathrm{min}^{-1}$ ). EMBS (estimated metabolic body size $)=$ fat-free mass $+17.7 \mathrm{~kg}$

Changes in M-low ( $\Delta$ M-low) and M-high ( $\Delta$ Mhigh) were both negatively related to $\Delta$ weight, $\Delta$ fat mass and $\Delta$ fat-free mass in both subjects with NGT and IGT (Fig. 1, Table 2). Of the variance in $\Delta \mathrm{M}$ low, $27 \%$ was explained by $\Delta$ weight. Additional determinants of $\Delta \mathrm{M}$-low, independent of $\Delta$ weight, were the baseline values of age, body weight, per cent body fat and M-low, explaining another $22 \%$, i. e. a total of $49 \%$ of the variance in $\Delta \mathrm{M}$-low.

Changes in AIR ( $\triangle$ AIR) and in the incremental 30-min insulin concentration ( $\Delta 30^{\prime}$-ins, OGTT) were both positively related to $\Delta$ weight, $\Delta$ fat mass and $\Delta$ fat-free mass in subjects with NGT but negatively in those with IGT (Fig. 1, Table 2). Thus, $\Delta$ weight alone was not a significant determinant of $\Delta$ AIR in the entire cohort but it became significant when the initial glucose tolerance status (NGT/IGT) was included into the model. The effect of $\Delta$ weight 
NGT $(n=151)$
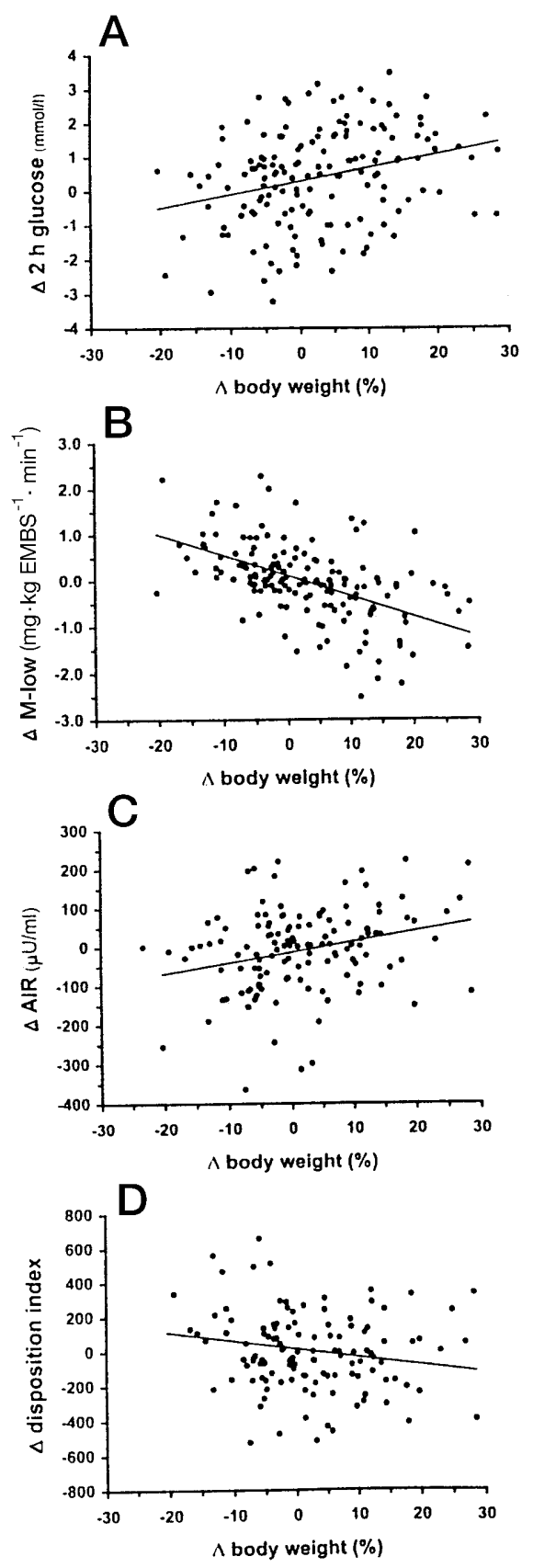

Fig. 1A-H. Relation of changes in 2-h glucose concentration, insulin action ( $\Delta \mathrm{M}$-low), insulin secretion ( $\Delta$ AIR) and in the disposition index to change in body weight ( $\Delta$ weight) in 209 Pima Indians with normal (NGT, A-D) or impaired (IGT, E-H) glucose tolerance, who either lost or gained weight over an average of $2.6 \pm 2.0$ years. AIR was assessed in 126 and 54 of the 151 and 58 subjects with NGT and IGT, respectively and multiplied with M-low to obtain the disposition index $\left(\mathrm{mU} \cdot \mathrm{mg} \cdot \mathrm{ml}^{-1} \cdot \mathrm{kgEMBS}{ }^{-1} \cdot \mathrm{min}^{-1}\right)$. A $r$ (Pearson's correlation coefficient) $=0.28, p<0.001, \mathbf{B} \quad r=-0.51, p<0.0001$, C, $r=0.26, p<0.01, \quad \mathbf{D} r=-0.18, p<0.05, \quad \mathbf{E} \quad r=0.67$, $p<0.0001, \mathbf{F} r=-0.54, p<0.0001, \mathbf{G} r=-0.51, p<0.0001$, $\mathbf{H} r=-0.56, p<0.0001$. EMBS (estimated metabolic body si$\mathrm{ze})=$ fat-free mass $+17.7 \mathrm{~kg}$
IGT $(n=58)$
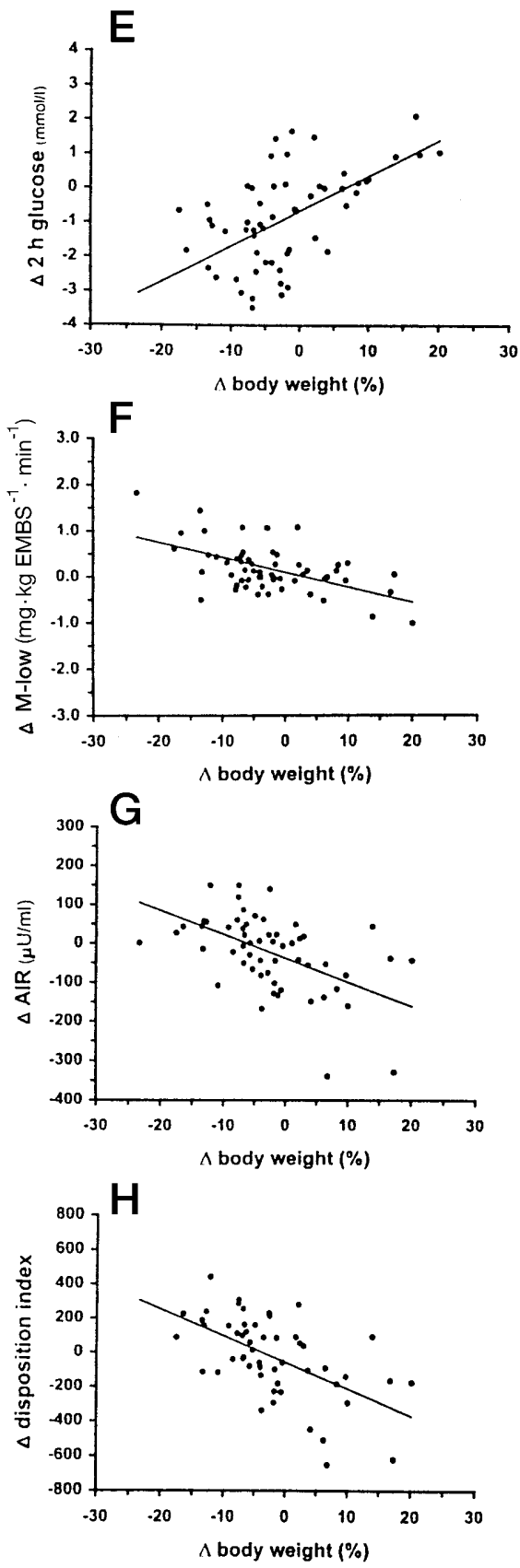

was no longer significant, however, when $\Delta$ M-low and baseline AIR were entered into the model. Together, these two factors explained $29 \%$ of the variance in $\triangle$ AIR.

To test whether there was an additional effect of sex, follow-up duration or the change in body fat distribution (waist-to-thigh ratio), we added these variables to the regression models but none was a significant determinant of any of the above changes.

Changes in the disposition index were negatively related to $\Delta$ weight, $\Delta$ fat mass, and $\Delta$ fat-free mass in both groups (Fig. 1, Table 2). The regression line for the correlation between $\Delta$ disposition index and 
Table 2. Relations between metabolic changes and changes in body weight, fat mass and fat-free mass over $2.6 \pm 2.0$ years in 209 Pima Indians with either normal (NGT) or impaired (IGT) glucose tolerance at baseline

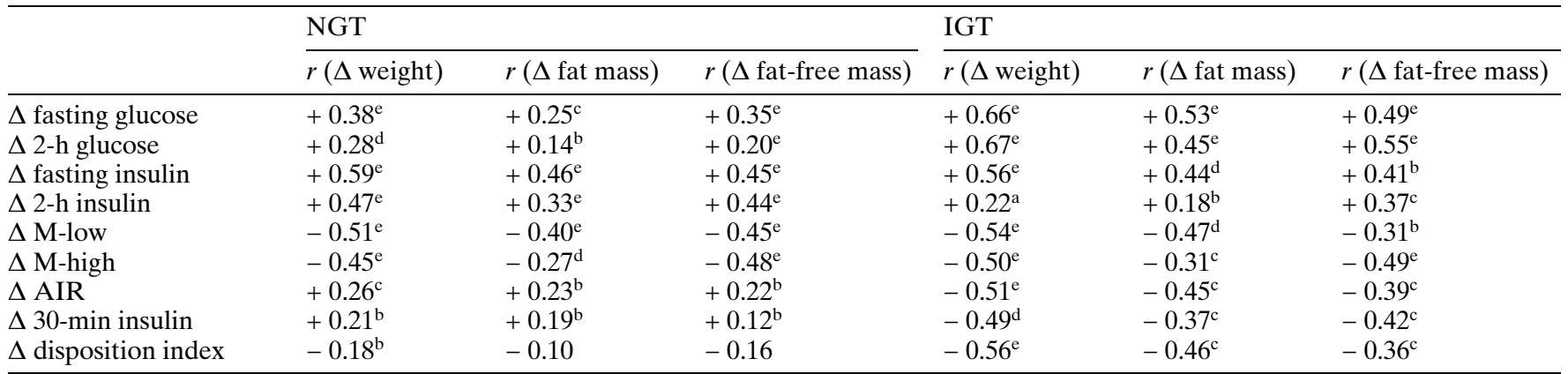

$r=$ Pearson correlation coefficient; ${ }^{\mathrm{a}} p<0.07,{ }^{\mathrm{b}} p<0.05$, ${ }^{\mathrm{c}} p<0.01,{ }^{\mathrm{d}} p<0.001,{ }^{\mathrm{e}} p<0.0001 ;$ M-low/M-high $=$ glucose disposal rates as assessed under low and high dose insulin infu-

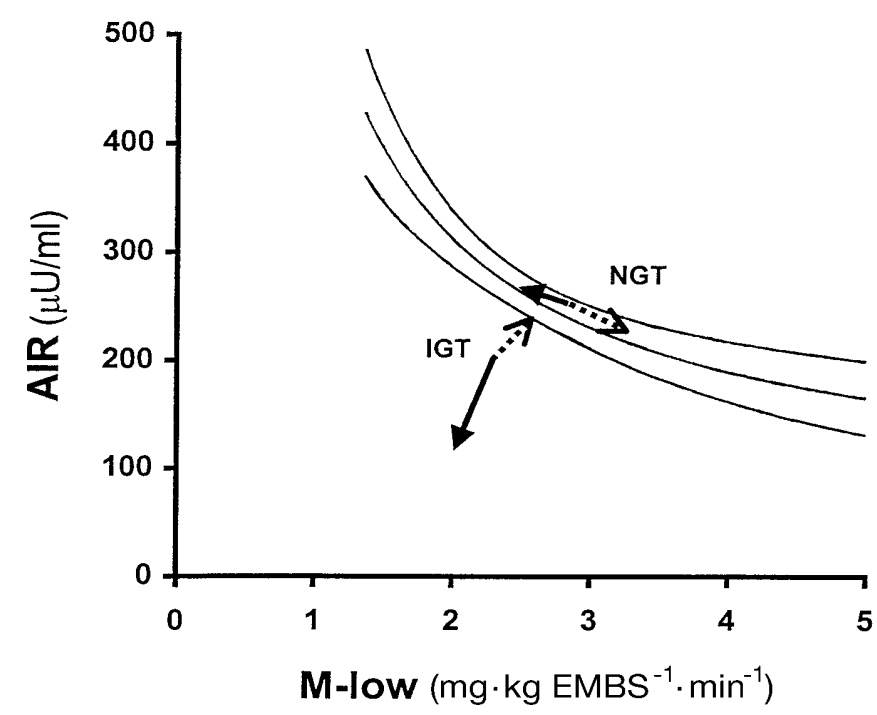

Fig. 2. Changes in insulin secretion (AIR) and insulin action (M-low) predicted for a $10 \%$ weight loss (dotted arrows) and $10 \%$ weight gain (solid arrows) in Pima Indians with either normal (NGT) or impaired (IGT) glucose tolerance. Changes in the 2 groups were plotted starting from the average values of AIR and M-low in subjects with NGT and IGT, respectively and superimposed onto the regression line and upper and lower $95 \%$ confidence intervals of the cross-sectional relation between AIR and M-low as assessed in 277 Pima Indians with NGT. Estimated metabolic body size $($ EMBS $)=$ fat-free mass $+17.7 \mathrm{~kg}$

$\Delta$ weight was steeper in the IGT than in the NGT group (Fig. 1).

Change in AIR was negatively related to $\Delta$ M-low in subjects with NGT $(r=-0.39, p<0.0001)$ but not related to $\Delta$ M-low in those with IGT $(r=0.10, \mathrm{NS})$. The vectors of the predicted changes in M-low and AIR for a $10 \%$ weight loss and $10 \%$ weight gain in subjects with NGT paralleled the slope of the regression line of the cross-sectional relation between these two measures (Fig. 2). In contrast, in subjects with IGT the vector of the predicted changes in M-low sions; AIR $=$ acute insulin response to a 25 -g i.v. glucose tolerance test; $30-\mathrm{min}$ insulin $=30-\mathrm{min}$ insulin response to an oral glucose tolerance test

and AIR with weight loss was directed towards the normal range, whereas that for the changes with weight gain was directed away from it (Fig. 2).

Effect of weight regain compared with weight maintenance. The physical characteristics and the anthropometric and metabolic changes in the three groups with either weight stability, weight loss/regain or weight loss/maintenance are given in Table 3 and Figure 3. All three groups included subjects with NGT and IGT and did not differ in the 2-h glucose concentration at baseline. Age, body weight and composition as well as all metabolic measures were similar among the three groups at baseline and there were no group differences in follow-up duration. The weight loss of $9.4 \pm 5.1 \%$ and $8.7 \pm 2.9 \%$ after 1.7 and 1.8 years in the weight loss/regain and weight loss/maintenance groups, respectively, was accompanied by significant and similar (time $\times$ group effect NS) reductions in fasting and 2-h glucose and insulin concentrations as well as by similar increases in $\mathrm{M}$ low $(+29$ and $+31 \%$, both $p<0.01)$, M-high $(+20$ and $+19 \%)$ and in the disposition index $(+29$ and $+21 \%$ ) whereas AIR did not change in either group (Table 3, Fig. 3). With subsequent weight regain, the initial improvements in fasting and $2-\mathrm{h}$ glucose and insulin concentrations as well as in M-low and Mhigh diminished and after 3.6 years, none of these variables differed from the initial values before weight loss (Table 3, Fig.3). In contrast, improvements in fasting and 2-h glucose and insulin concentrations as well as in M-low, M-high and in the disposition index after weight loss were largely preserved over 3.3 years with weight maintenance (Table 3 , Fig. 3). No changes in any of the metabolic variables occurred in the control subjects, who remained weight stable over a similar period and the overall time effect for all anthropometric and metabolic variables except for AIR differed significantly among the three groups (time $\times$ group effect $p<0.05$ ). 

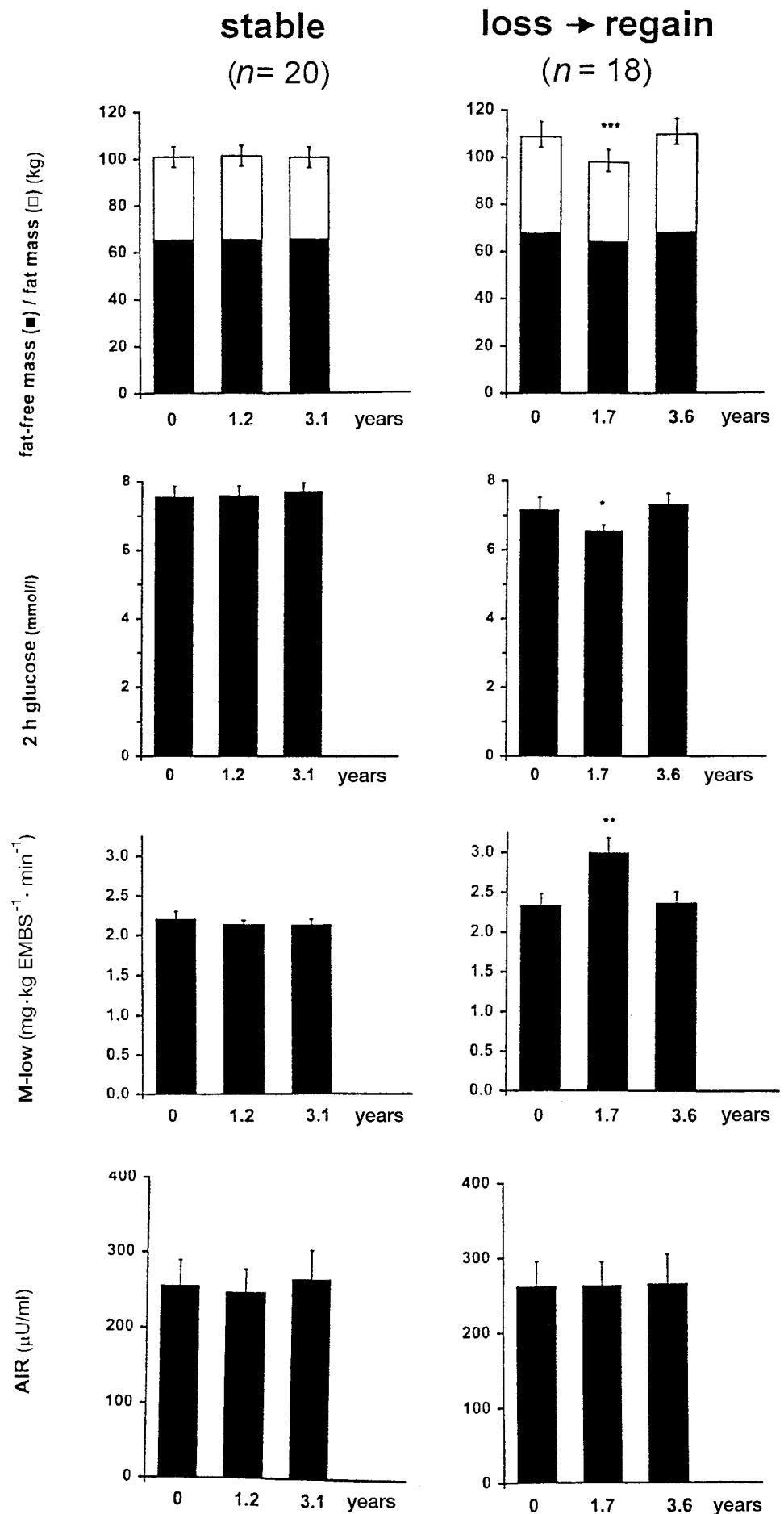
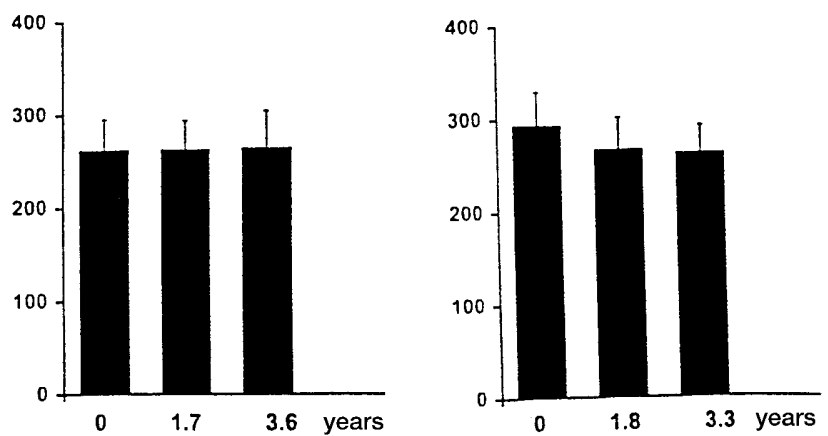

Fig. 3. Changes in body weight and composition, 2-h glucose concentration, insulin action (M-low) and insulin secretion (AIR) in 53 non-diabetic Pima Indians who were either weight stable (left panels, $n=20$ ) or lost at least $5 \%$ of initial body weight and then subsequently either regained (middle panel, $n=18$ ) or maintained (right panel, $n=15$ ) weight over an average of $3.1,3.6$ and 3.3 years, respectively $(* \mathrm{p}<0.05$, $* * \mathrm{p}<0.01, * * * \mathrm{p}<0.001$ compared with baseline)
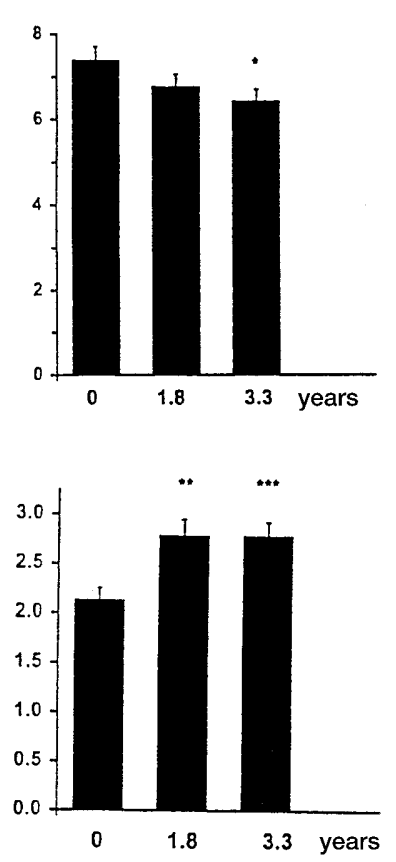

\section{loss $\rightarrow$ maintain}

$$
(n=15)
$$

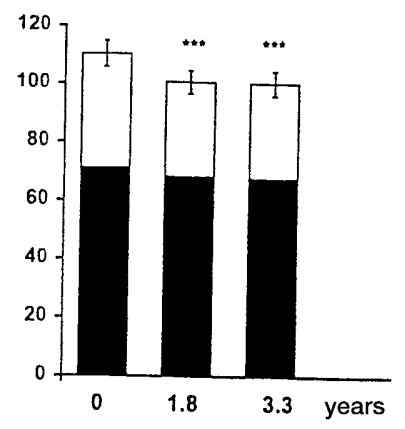

\section{Discussion}

Obese people have an increased risk for Type II diabetes [1-3]. Weight gain in adulthood is an additional risk factor for diabetes, independent of obesity [3-5] whereas profound weight reduction in morbidly obese patients after bariatric surgery can substantially reduce the risk of developing diabetes [41]. Intervention studies indicate that even a moderate reduction in body weight can, at least in the short term, lead to improvements in obesity-associated metabol- 
Table 3. Physical characteristics and metabolic changes in 53 non-diabetic Pima Indians who were either weight stable or lost at least $5 \%$ body weight and subsequently either regained or maintained body weight over an average of $3.3 \pm 1.4$ years

\begin{tabular}{|c|c|c|c|c|c|c|c|c|c|}
\hline & \multicolumn{3}{|c|}{ Weight stable } & \multicolumn{3}{|c|}{ Weight loss/regain } & \multicolumn{3}{|c|}{ Weight loss/maintenance } \\
\hline & study 1 & study 2 & study 3 & study 1 & study 2 & study 3 & study 1 & study 2 & study 3 \\
\hline$n$ & 20 & & & 18 & & & 15 & & \\
\hline NGT/IGT & $11 / 9$ & $11 / 9$ & $12 / 8$ & $10 / 8$ & $16 / 2$ & $12 / 6$ & $10 / 5$ & $11 / 4$ & $11 / 4$ \\
\hline age (y) & $28.2 \pm 4.7$ & $29.4 \pm 4.7$ & $31.3 \pm 4.6$ & $26.6 \pm 5.2$ & $28.3 \pm 5.4$ & $30.2 \pm 5.0$ & $28.1 \pm 5.6$ & $29.9 \pm 5.9$ & $31.4 \pm 5.9$ \\
\hline fasting glucose $(\mathrm{mmol} / \mathrm{l})$ & $5.1 \pm 0.4$ & $5.1 \pm 0.5$ & $5.0 \pm 0.3$ & $5.4 \pm 0.6$ & $4.9 \pm 0.4^{\mathrm{a}}$ & $5.2 \pm 0.5$ & $5.3 \pm 0.6$ & $5.0 \pm 0.6^{\mathrm{a}}$ & $5.0 \pm 0.3^{\mathrm{a}}$ \\
\hline 2-h insulin $(\mu \mathrm{U} / \mathrm{ml})$ & $231 \pm 144$ & $236 \pm 167$ & $250 \pm 157$ & $183 \pm 139$ & $128 \pm 90^{\mathrm{a}}$ & $203 \pm 113$ & $192 \pm 151$ & $143 \pm 73^{a}$ & $148 \pm 100^{\mathrm{a}}$ \\
\hline 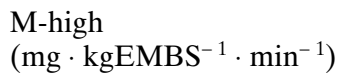 & $7.9 \pm 2.1$ & $8.2 \pm 2.8$ & $8.3 \pm 1.8$ & $8.3 \pm 1.9$ & $10.0 \pm 1.7^{\mathrm{b}}$ & $8.6 \pm 2.0$ & $8.1 \pm 2.1$ & $9.7 \pm 2.7^{\mathrm{b}}$ & $9.3 \pm 2.0^{\mathrm{b}}$ \\
\hline disposition index & $557 \pm 310$ & $515 \pm 280$ & $581 \pm 383$ & $587 \pm 364$ & $759 \pm 499^{a}$ & $605 \pm 433$ & $594 \pm 429$ & $717 \pm 508^{a}$ & $730 \pm 534^{\mathrm{a}}$ \\
\hline
\end{tabular}

Means \pm SD. The superscripts indicate significant differences compared to baseline (paired $t$ test ${ }^{\mathrm{a}} p<0.05,{ }^{\mathrm{b}} p<0.01$ ) within each group. Results of the group comparison are given in the text

ic disturbances such as impaired glucose tolerance, hyperinsulinaemia and insulin resistance [9-21].

In the present observational study, we analysed longitudinal data from a large number of Pima Indians, a population with a very high prevalence of obesity and Type II diabetes, to examine and quantify the relation between long-term changes in body weight and changes in insulin action and insulin secretion. Our results show several clinically relevant findings.

In the first part of our study, we found that over a wide range of weight changes, changes in insulin action over 2.7 years were linearly related to the amount of weight change. The finding of a negative relation between changes in insulin action and changes in body weight agrees with that of two previous intervention studies [10,42], which showed similar correlations over a shorter period. In a more recent 4-month dietary intervention study in 32 obese subjects [9], improvements in insulin action were not found to be related to the overall amount of weight loss but instead specifically related to the loss in visceral adipose tissue as determined by computed tomography. Similar findings have been reported for the improvements in plasma glucose and lipid concentrations with weight loss [43]. In our study, the changes in waist circumference or waist-to-thigh ratio, less precise measures of abdominal body fat accumulation than obtained from tomograms, were not statistically significant independent determinants of the change in insulin action, suggesting that a loss of body weight in itself can improve insulin action. It could also be hypothesized that the decrease in insulin action with weight gain is more closely related to the increase in fat mass than in fat-free mass as skeletal muscle is the major contributor to whole body glucose disposal in humans. This was not the case, however. Instead, in multiple regression analyses, the change in insulin action was more closely related to the change in weight than to the change in fat mass or fat-free mass. In this respect, it is important to consider that changes in total body weight can be measured much more precisely than changes in fat mass and fatfree mass. Moreover, body weight can increase considerably in a subject with only minor changes in adiposity (percent body fat), i. e., when fat mass and fat-free mass increase proportionately. Our statistical analysis also showed that the deterioration in insulin action with weight gain is similar in magnitude to the improvement in insulin action associated with weight loss. Finally, we found that the negative relation between changes in insulin action and changes in body weight was present in subjects with normal (NGT) and impaired (IGT) glucose tolerance with no significant difference between the two groups.

In contrast, we found that subjects with NGT and IGT differ substantially in their insulin secretory response to weight change in that the changes in both the acute insulin response (AIR) to intravenous glucose and the 30-min insulin response to oral glucose were positively related to weight change in subjects with NGT but negatively in those with IGT. To illustrate how the insulin secretory response to weight change differs between people with NGT compared with IGT relative to the simultaneous change in insulin action, we plotted the changes in AIR predicted for a $10 \%$ weight loss and $10 \%$ weight gain in each group against the predicted changes in M-low (Fig.2). These plots were superimposed onto the plot of the regression line and upper and lower limits of the $95 \%$ confidence interval of the cross-sectional relation between AIR and M-low, as determined in 277 Pima Indians with NGT (Fig. 2). The relation between insulin secretion and insulin action is known to be hyperbolic $[22,29]$, i. e. cross-sectionally, insulin secretion increases with decreasing insulin action and 
vice versa. As can be inferred from Figure 2, the changes in insulin secretion with weight changes in subjects with NGT are physiologically meaningful in that an increase or decrease in insulin secretion with weight gain or weight loss, respectively compensates for the simultaneous decrease/increase in insulin action. That this was indeed the case was confirmed by the positive correlation between changes in insulin secretion and changes in insulin action in subjects with NGT, a finding that agrees with that of a weight loss intervention study in obese normal glucose tolerant Caucasians [11]. In contrast, in Pima Indians with IGT, insulin secretion failed to increase in response to the decrease in insulin action with weight gain and the changes in insulin secretion and insulin action were unrelated. Instead, insulin secretion decreased with weight gain in subjects with IGT, in parallel to a decrease in insulin action (Fig.2). These differential responses to weight change in subjects with NGT compared with IGT became most apparent in the disposition index, an integrated measure of insulin action and insulin secretion originally derived from the minimal model [22]. According to the "hyperbolic law of glucose tolerance" [22], this index should remain constant as long as changes in insulin action are compensated for by changes in insulin secretion to maintain glucose homeostasis. In fact, there was only a slight decrease in the disposition index with weight gain in subjects with NGT and, presumably as a result, only a relatively small increase in the 2-h glucose concentration. In contrast, in subjects with IGT, the disposition index decreased considerably with weight gain and, in turn, there was a steeper increase in the 2-h glucose concentration. This decrease in the disposition index and increase in the 2-h glucose concentrations with weight gain in the IGT group would have been even greater, if subjects with diabetes at follow-up were included. We decided, however, to exclude these subjects because overt hyperglycaemia is known to have detrimental effects on both insulin action and insulin secretion independent of weight changes. Our results also suggest that subjects with IGT could especially benefit from weight loss, which can simultaneously improve insulin action and insulin secretion. Our observational data do not allow us to uncover the exact physiological mechanism(s) responsible for the differential insulin secretory responses to weight changes in subjects with NGT compared with IGT. It seems reasonable, however, that subtle defects in beta-cell secretory capacity, either inherited or acquired, prevented an adequate increase in insulin secretion to compensate for the decrease in insulin action in subjects with IGT [29].

The most appropriate method for the in vivo assessment of insulin action and insulin secretion is a matter of debate. It is noteworthy, therefore, that our findings were consistent, irrespective of whether insulin action and insulin secretion were measured under physiological (M-low, 30-min insulin response to oral glucose) or supra-physiological (M-high, acute insulin response to i.v. glucose) conditions.

An important observation from our analyses is the high interindividual variability in the insulin action and insulin secretory responses to weight changes. The long-term metabolic effects of weight loss and weight gain seemed to differ considerably among subjects, particularly in the range of minor weight changes $(-5$ to $+5 \%)$. In our multiple regression analyses, we were able to identify several factors that explained part of the variance in changes in glucose tolerance, M-low and AIR, in addition to the effect of weight change. In total, only about $50 \%$ and $30 \%$ of the variance in $\Delta$ M-low and $\Delta$ AIR could, however, be explained. Because not all of the remaining variance can be attributed to the variability of the methods (e.g. only $\sim 15 \%$ for the hyperinsulinaemic clamp, [44]), it seems that other factors are involved that could in part be genetically determined. The above findings could have several important clinical implications. Firstly, our data indicate that even modest amounts of weight change over several years can affect glucose tolerance and insulin action. It also seems, however, that this does not necessarily have to be the case, as responses vary substantially among subjects. Secondly, our data indicate that improvements and deteriorations in glucose tolerance and insulin action increase in proportion to the amount of weight loss and gain, respectively. Thus, we found no evidence of a threshold above which further weight loss has no further beneficial effect on insulin action as has recently been suggested based on results of a short-term intervention study [9].

In the second part of our study, we aimed to examine the long-term outcome of improved glucose tolerance and insulin action after weight loss, specifically in response to weight regain compared with successful weight maintenance. Our results indicate that the improvements in insulin action and glucose tolerance after an average weight loss of approximately $10 \%$ can be largely preserved over several years if the reduction in body weight is successfully maintained. This finding is important because the improvement in insulin action found after short-term weight loss interventions could in part be a temporary consequence of a negative energy balance, rather than a sustained effect of the weight loss itself [11]. We found no statistically significant change in insulin secretion with weight loss and subsequent weight regain or maintenance. This finding is not surprising, however, given that both groups comprised of subjects with NGT and IGT who had inverse insulin secretory responses to weight changes. Unfortunately, the limited number of subjects in this second part of our study precluded any meaningful subgroup analysis for subjects with NGT compared with IGT. 
It is well known that successful weight maintenance after weight loss is achieved by only a small number of people and that the majority will subsequently regain most of the weight [45]. It has been suggested that repeated weight loss and weight regain has adverse effects on body fat accumulation [24, 26], glucose tolerance [26] and risk of diabetes [27], although the postulated health risk of such "weight cycling" is a matter of debate [46-49]. Our results do not suggest that weight cycling has detrimental effects on insulin action, insulin secretion or glucose tolerance because all these measures were similar to baseline after a single bout of weight loss and subsequent weight regain. This agrees with previous epidemiological findings in Pima Indians that weight fluctuation is not associated with increased diabetes risk in this population [5]. Fasting and 2-h insulin concentrations also reverted back to baseline values, a finding that agrees with those of a previous intervention study [42]. Because of the long follow-up duration, it might be possible that the results of our weight maintenance/regain analysis were confounded by ageing. To control for this possible effect, we included a weight stable control group, in which no changes in glucose tolerance and insulin action occurred over a similar period. Together, the findings that glucose tolerance and insulin action can be improved in the long term with sustained weight loss and are not negatively affected by weight loss and regain provide further arguments to support the recommendation of weight loss in obese people at risk for diabetes, even if many of them may not achieve long-term benefits.

Our study was observational, not interventional, which has both disadvantages and advantages. On one hand, it cannot be assumed that the weight loss was intentional. It is unlikely that illness was a cause of weight loss, however, because most participants in our longitudinal study maintain close contact with the clinical research centre and undergo a comprehensive medical evaluation before each admission. It is also possible that some of the weight gainers/losers in our study had gained/lost even more weight but had partially reverted to their initial weight before the follow-up admission. Besides these putative limitations, the observational design of our study offers several potential advantages over previous intervention studies. These include the long follow-up duration of several years, highly relevant to the longterm management of obesity and diabetes. Furthermore, the relatively gradual weight changes over several years in our study probably resembles more closely the natural course of body weight changes in obese subjects than the drastic weight reduction often induced in dietary intervention studies.

In conclusion, the results of our longitudinal analyses show in a large number of subjects that improvements in insulin action with long-term weight loss are proportional to the amount of weight loss, similar in magnitude to the impairment in insulin action associated with weight gain, preserved with weight maintenance and similar between subjects with NGT and IGT. Weight gain seems, however, to have more detrimental effects in subjects with IGT in whom insulin secretion fails to increase to compensate for the decreased insulin action.

Acknowledgements. We gratefully acknowledge Mr. M. Milner, Mrs. C. Massengill and the nurses of the Clinical Research Unit as well as Dr. A. Salbe and the staff of the metabolic kitchen for their care of the patients in the study and the Clinical Diabetes and Nutrition Section technical staff for assisting in the laboratory analyses. We would like to thank the members and leaders of the Gila River Indian Community, without whose continuing cooperation this study would not have been possible.

\section{References}

1. Colditz GA, Willett WC, Stampfer MJ et al. (1990) Weight as a risk factor for clinical diabetes in women. Am J Epidemiol 132: 501-513

2. Knowler WC, Pettitt DJ, Saad MF, Bennett PH (1990) Diabetes mellitus in the Pima Indians: Incidence risk factors and pathogenesis. Diabetes Metab Rev 6: 1-27

3. Chan JM, Rimm EB, Colditz GA, Stampfer MJ, Willett WC (1994) Obesity, fat distribution, and weight gain as risk factors for clinical diabetes in men. Diabetes Care 17: 961-969

4. Ford ES, Williamson DF, Liu S (1997) Weight change and diabetes incidence: findings from a national cohort of US adults. Am J Epidemiol 146: 214-222

5. Hanson RL, Narayan KMV, McCance DR et al. (1995) Rate of weight gain, weight fluctuation, and incidence of NIDDM. Diabetes 44: 261-266

6. Bogardus C (1996) Metabolic abnormalities in the development of non-insulin dependent diabetes mellitus. In: Le Roith D, Taylor SI, Olefwski JM (eds) Diabetes Mellitus. Lippincot-Raven Publishers Philadelphia, PA

7. Ferrannini E, Camastra S (1998) Relationship between impaired glucose tolerance, non-insulin-dependent diabetes mellitus and obesity. Eur J Clin Invest 28 [Suppl.2]:3-7

8. Weyer C, Bogardus C, Pratley RE (1999) Metabolic characteristics of individuals with impaired fasting glucose and/or impaired glucose tolerance. Diabetes 48: 2197-2203

9. Goodpasture BH, Kelley DE, Wing RR, Meier A, Thaete FL (1999) Effects of weight loss on regional fat distribution and insulin sensitivity in obesity. Diabetes 48: 839-847

10. Muscelli E, Camastra S, Catalano C et al. (1997) Metabolic and cardiovascular assessment in moderate obesity: effect of weight loss. J Clin Endocrinol Metab 82: 2937-2943

11. Marcovic TP, Jenkins AB, Campbell LV, Furler SM, Kraegen EW, Chisholm DJ (1998) The determinants of glycemic responses to diet restriction and weight loss in obesity and NIDDM. Diabetes Care 21: 687-694

12. Bryson JM, King SE, Burns CM, Baur LA, Swaraj S, Caterson ID (1996) Changes in glucose and lipid metabolism following weight loss produced by a very low calorie diet in obese subjects. Int J Obes 20: 338-345

13. Ahmad F, Considine RV, Ohannesian JP, Marco CC, Goldstein BJ (1997) Improved sensitivity to insulin in obese subjects following weight loss is accompanied by reduced protein-tyrosine phosphatases in adipose tissue. Metabolism 46: $1140-1145$ 
14. Webber J, Donaldson M, Allison SP, Fukagawa NK, Macdonald IA (1994) The effect of weight loss in obese subjects on the thermogenic, metabolic and haemodynamic responses to the glucose clamp. Int J Obes 18: 725-730

15. Calle-Pascual AL, Saavedra A, Benedi A et al. (1995) Changes in nutritional pattern, insulin sensitivity and glucose tolerance during weight loss in obese patients from a Mediterranean area. Horm Metab Res 27: 499-502

16. Niskanen L, Uusitupa M, Sarlund H, Siitonen O, Paljärvi L, Laakso M (1996) The effects of weight loss on insulin sensitivity, skeletal muscle composition and capillary density in obese non-diabetic subjects. Int J Obes 20: 154-160

17. Golay A, Felber JP, Dusmet M, Gomez F, Curchod B, Jequier E (1985) Effect of weight loss on glucose disposal in obese and obese diabetic subjects. Int J Obes 9: 181-190

18. Fletcher JM, McNurlan MA, McHardy KC (1989) Residual abnormalities of insulin secretion and sensitivity after weight loss by obese women. Eur J Clin Nutr 43: 539-545

19. Letiexhe MR, Scheen AJ, Gerard PL, Desaive C, Lefebvre PJ (1994) Insulin secretion, clearance and action before and after gastroplasty in severely obese subjects. Int J Obes 18: 295-300

20. Letiexhe MR, Scheen AJ, Gerard PL, Desaive C, Lefebvre PJ (1995) Postgastroplasty recovery of ideal body weight normalizes glucose and insulin metabolism in obese women. J Clin Endocrinol Metab 80: 364-369.

21. Wing RR, Koeske R, Epstein LH et al. (1987) Long-term effects of moderate weight loss in type 2 diabetic patients. Arch Int Med 14: 1749-1753

22. Bergman RN (1989) Toward physiological understanding of glucose tolerance: minimal model approach. Diabetes 38: $1512-1527$

23. Ferrannini E (1998) Insulin resistance vs. insulin deficiency in non-insulin dependent diabetes mellitus: problems and prospects. Endocr Rev 19: 477-490

24. Jen K-LC, Lu H, Savona L, Watkins A, Shaw M (1995) Long-term weight cycling reduces body weight and fat free mass, but not fat mass in female Wistar rats. Int J Obesity 19: 699-708

25. Rodin J, Radke-Sharpe N, Reduffe-Scrive M, Greenwood MRC (1990) Weight cycling and fat distribution. Int J Obes 14: 303-310

26. Lissner L, Andres R, Muller DC, Shimokata H (1989) Body weight variability in men: metabolic rate, health and longevity. Int J Obes 14: 373-383

27. Holbrook TL, Barrett-Connor C, Wingard DL (1989) The association of lifetime weight and weight control patterns with diabetes among men and women in an adult community. Int J Obes 13: 723-729

28. Weyer C, Bogardus C, Pratley RE (1999) Metabolic factors contributing to increased resting metabolic rate and decreased insulin-induced thermogenesis during the development of type 2 diabetes. Diabetes 48: 1607-1614

29. Weyer C, Bogardus C, Mott DM et al. (1999) The natural history of insulin secretory dysfunction and insulin resistance in the pathogenesis of type 2 diabetes mellitus. J Clin Invest 104: 787-794

30. World Health Organization (1985) Diabetes mellitus: Report of a WHO study group. WHO Technical Report Series 17: 45-51 World Health Organisation, Geneva
31. American Diabetes Association (1998) Report of the expert committee on the diagnosis and classification of diabetes mellitus (Committee Report). Diabetes Care 21:S5-S19

32. Goldman RF, Buskirk ER (1961) A method for underwater weighing and the determination of body density. In: Brozek J, Herschel A (eds) Techniques for measuring body composition. National Academy of Sciences Washington DC pp 78-106

33. Mazess RB, Barden HS, Bisek JP, Hanson J (1990) Dualenergy $\mathrm{x}$-ray absorptiometry for total-body and regional bone-mineral and soft-tissue composition. Am J Clin Nutr 51: $1106-1112$

34. Siri WE (1961) Body composition from fluid spaces and density: analysis of methods. In: Brozek J, Herschel A (eds) Techniques for measuring body composition. National Academy of Sciences Washington DC pp 223-244

35. Tataranni PA, Ravussin E (1995) Use of dual-energy x-ray absorptiometry in obese individuals. Am J Clin Nutr 62: 730-734

36. Herbert Y, Lau K, Gottlieb CW, Bleicher SJ (1965) Coated charcoal immunoassay of insulin. J Clin Endocrinol Metab 25: $1375-1384$

37. Yalow RS, Berson SA (1960) Immunoassay of endogenous plasma insulin in man. J Clin Invest 39: 1157-1167

38. Lillioja S, Bogardus C (1988) Obesity and insulin resistance: lessons learned from the Pima Indians. Diabetes Metab Rev 4: 517-540

39. Schwartz MW, Boyko EJ, Kahn SE, Ravussin E, Bogardus C (1995) Reduced insulin secretion: an independent predictor of body weight gain. J Clin Endocrinol Metab 80: $1571-1576$

40. SAS Institute Inc. (1990) SAS/STAT User's Guide. Version 6 , (4 $4^{\text {th }}$ ed. $)$ Cary, NC

41. Long SD, O'Brien K, MacDonald KG Jr et al. (1994) Weight loss in severly obese subjects prevents the progression of impaired glucose tolerance to Type II diabetes. A longitudinal intervention study. Diabetes Care 17: 372-375

42. Wing RR (1997) Insulin sensitivity as a predictor of weight regain. Obes Res 5: 24-29

43. Fujioka S, Matsuzawa Y, Tokuaga K et al. (1991) Improvement of glucose and lipid metabolism associated with selective reduction of intra-abdominal visceral fat in premenopausal women with visceral fat obesity. Int $\mathbf{J}$ Obes 15: 853-859

44. Bogardus C, Lillioja S, Mott DM, Hollenbeck C, Reaven G (1987) Relationship between degree of obesity and in vivo insulin action in man. Am J Physiol 248:E286-E291

45. Wadden T (1989) Treatment of obesity by moderate and severe caloric restriction. Results of clinical research trials. Ann Intern Med 119: 688-692

46. National Task Force on the Prevention and Treatment of Obesity (1995) Weight cycling. JAMA 272: 1196-1202

47. Brownell KD, Rodin J (1994) Medical, metabolic, and psychological effects of weight cycling. Arch Intern Med 154: 1325-1330

48. Podar T, Solntsev A, Väli M, Vinogradova T, Podar I (1996) No deterioration of glucose tolerance in weight cycling obese. Int J Obes 20: 921-924

49. Van der Kooy K, Leenen R, Seidell JC, Deurenberg P, Hautvast JG (1993) Effect of a weight cycle on visceral fat accumulation. Am J Clin Nutr 58: 853-857 